\title{
КОМПЬЮТЕРНОЕ МОДЕЛИРОВАНИЕ ДИНАМИКИ МНОГОЗВЕННЫХ МАНИПУЛЯТОРОВ С УПРАВЛЕНИЕМ
}

\section{COMPUTER SIMULATION OF THE DYNAMICS OF MULTI- LINK MANIPULATORS WITH CONTROL}

S. Ivanov

T. Zudilova

I. Osetrova

T. Voytyuk

Summary. The problem of mathematical modeling of the dynamics of manipulators with control by means of a software module developed and registered by the authors in functional programming language is considered. A universal software module allows you to build and analyze a model of various types of manipulators up to six degrees of mobility, presented in the form of a system of nonlinear differential equations. The solution of the studied model is constructed by the numerical RungeKutta method and the analytical method of polynomial transformations. The paper describes the main blocks of the program on the example of the analysis of a software-controlled manipulator with three degrees of mobility. The software module can be used for engineering analysis of various types of manipulators.

Keywords: computer modeling, dynamics of manipulators, analysis of manipulators, software module, mathematical modeling.

\author{
Иванов Сергей Евгеньевич \\ К.ф.--м.н., дочент, НИУ ИТМО (Санкт-Петербург) \\ sivanov@mail.ifmo.ru \\ Зудилова Татьяна Викторовна \\ К.т.н., дочент, НИУ ИТМО (Санкт-Петербург) \\ zudilova@ifmo.spb.ru \\ Осетрова Ирина Станиславовна \\ Cm. преподаватель, НИУ ИТМО (Санкт-Петербург) \\ irina@ifmo.spb.ru \\ Войтюк Татьяна Евгеньевна \\ К.т.н., дочент, НИУ ИТМО (Санкт-Петербург) \\ taire2006@yandex.ru
}

Аннотация. Рассматривается задача математического моделирования динамики манипуляторов с управлением посредством разработанной и зарегистрированной авторами программного модуля на языке функционального программирования. Универсальный программный модуль позволяет построить и провести анализ модели различных видов манипуляторов до шести степеней подвижности, представленных в форме системы нелинейных дифференциальных уравнений. Решение исследуемой модели строится численным методом Рунге-Кутты и аналитическим методом многочленных преобразований. В работе приведено описание основных блоков программы на примере анализа программно-управляемого манипулятора с тремя степенями подвижности. Программный модуль может применяться для инженерного анализа различных видом манипуляторов.

Ключевые слова: компьютерное моделирование, динамика манипуляторов, анализ манипуляторов, программный модуль, математическое моделирование.

скоростей, ускорений и траекторий движения манипулятора, блок управления манипулятором.

Решение нелинейной системы дифференциальных уравнений динамики строится численным методом Рунге-Кутты и аналитическим методом преобразований.

Множество современных научных работ посвящено задачам анализа многозвенных манипуляторов. В работах [2-4] исследуется промышленный робот - манипулятор KUKA youBot. В работе [5] проводится математическое и компьютерное моделирование вращающегося манипулятора и четырехколесного мобильного манипулятора. В работе [6] выполняется моделирование роботов-манипуляторов типа «хобот». В отличие от приведенных работ разработанный программный модуль позволяет исследовать различные виды многозвенных манипуляторов. 
Рассмотрим блоки программного модуля на примере анализа программно-управляемого трехзвенного манипулятора с тремя степенями подвижности.

Предполагаем, что управляющее воздействие для приводов манипулятора имеет кусочно-гладкую линейную форму и схват манипулятора равномерно движется с постоянной рабочей скоростью.

Кинематическая схема трехзвенного манипулятора состоит из трех вращательных кинематических пар.

В блоке сборки уравнений кинематики матричным методом для описания кинематики вводим три относительные системы координат, связанные с тремя звеньями робота. Абсолютная система координат связана с неподвижным основанием манипулятора. Углы поворотов звеньев манипулятора примем за обобщенные координаты. В блоке определяются матрицы перехода из текущей в последующую систему координат. Для матрицы перехода из абсолютной системы координат в последнюю систему координат для схвата выполняется перемножение всех промежуточных матриц перехода. В результате получаем зависимость абсолютных декартовых координат схвата манипулятора $x, y, z$ от трех обобщенных координат $q 1, q 2, q 3$ в форме трех алгебраических равенств.

В блоке решения системы уравнений кинематики выполняется решение системы трех алгебраических уравнений. В результате получаем зависимость трех обобщенных координат $q 1(x, y, z), q 2(x, y, z), q 3(x, y, z)$ от координат схвата трехзвенного манипулятора.

При условии равномерного движения схвата трехзвенного манипулятора с постоянной рабочей скоростью $v$ выполняются равенства $x=v 1 t, y=v 2 t, z=v 3 t$. Здесь $v 1, v 2, v 3$ проекции рабочей скорости на оси $x$, $y, z$.

Подставив равенства для равномерного движения в функции обобщенных координат получим зависимости обобщенных координат трехзвенного манипулятора от времени $t: q 1(t), q 2(t), q 3(t)$. Продифференцировав по времени функции обобщенных координат, получим обобщенные скорости трехзвенного манипулятора: $q 1$ ' $(t), q 2$ ' (t), q3' (t). Продифференцировав по времени функции обобщенных скоростей, получим обобщенные ускорения трехзвенного манипулятора: q1 "' (t), q2” (t), $q 3 "(t)$.

В блоке сборки уравнений динамики посредством матричных уравнений Лагранжа вычисляется суммарная кинетическая и потенциальная энергии всех звеньев манипулятора. Подставив в матричные уравнения Ла- гранжа, получаем систему уравнений движения манипулятора. В правых частях уравнений Лагранжа содержаться моменты от управляющих сил. Система уравнений динамики трехзвенного манипулятора представлена нелинейной системой дифференциальных уравнений второго порядка вида:

$$
(a 0+a 1 \cos (q 1)) q 1 "+a 2 q 1 \text { ' } 22 \sin (q 2)+a 3
$$

$\cos (q 1)+a 4 \cos (q 1+q 2)=a 5 F 1$,

$$
b 0 q 2 "+b 1 q 1 \text { ' } q 1 \text { 'sin }(q 2)+b 2 \cos (q 2)+b 3
$$

$\cos (q 1+q 2)+b 4=b 5 F 2$,

$c 0 q 3 "+c 2 q 3+c 3=c 4 F 3$,

где $F 1(t), F 2(t), F 3(t)-$ функции управляющих сил, $a i, b i, c i-$ постоянные.

В блоке решения нелинейной системы дифференциальных уравнений динамики выполняется решение численным методом Рунге-Кутты. Для аналитического решения системы нелинейных дифференциальных уравнений применяются различные методы: линеаризации, усреднения, малого параметра, гармонического баланса, методом многочленных преобразований [7]. Методом многочленных преобразований получено аналитическое решение системы дифференциальных уравнений. Для построения аналитического решения вначале проводят нормализацию системы для приведения нелинейных коэффициентов системы к малым величинам.

В блоке управления манипулятором определяются параметры для моментов от управляющих сил в зависимости от обобщенных координат. Управляющее воздействие представляет собой силу, момент создаваемую приводами звеньев. Система управления приводом регулирует момент двигателя. Для управления приводом необходимо найти управляющее воздействие, которое обеспечивает движение манипулятора по заданной траектории. Подставив в систему уравнений движения функции обобщенных координат, скоростей и ускорений, получим функции управляющих сил. Определено управляющее воздействие от приводов в форме линейной функции от времени.

В случае представления управляющего воздействия линейными функциями времени $F(t)=p 1+p 2 t$, коэффициенты $p 1, p 2$ являются параметрами управления. Для проверки управляющих воздействий приводов при заданных параметрах управления определяется численное решение системы дифференциальных уравнений методом Рунге-Кутты.

В блоке построение графиков координат, скоростей, ускорений и траекторий движения манипулятора выполняется графическое представление результатов, полученных численным и аналитическим методом. 


\section{Зак^ючение}

Разработанный универсальный программный модуль позволяет проводить исследования динамики манипуляторов различных кинематических схем со многими степенями подвижности. Исследуемые математические модели представлены в форме системы нелинейных дифференциальных уравнений. Решение исследуемой модели строится численным методом Рунге-Кутты и аналитическим методом многочленных преобразований. Программный модуль может применяться для инженерного анализа различных видом манипуляторов.

\section{ЛИТЕРАТУРА}

1. Иванов С. Е. Программа анализа динамических моделей полиномиальной структуры для манипуляторов // Свидетельство № 2018619899 РФ, 2018

2. Капустина 0. М. Анализ сингулярных конфигураций мобильного манипулятора KUKA youBot // Вестник МЭИ. 2019. № 1. С. 93-97.

3. Адамов Б. И., Капустина 0. М., Меркурьев И. В., Панкратьева Г. В. Кинематика, динамика и управление движением мобильного робота-манипулятора KUKA youBot. - Изд-во МЭИ Москва, 2018. - 72 с.

4. Капустина 0. М. Манипулятивность робота KUKА уоuBot и планирование его движений // Extreme Robotics. — 2018. — T. 1.—№ 1. С. $286-301$.

5. Красинский А. Я. Математическое и компьютерное моделирование манипуляторов с нелинейной геометрической связью // Инженерный журнал: наука и инновации. 一 2018. - № . 4 (76).

6. Каганов Ю.Т., Карпенко А. П. Математическое моделирование кинематики и динамики робота-манипулятора типа «хобот». 1. Математические модели секции манипулятора, как механизма параллельной кинематики типа «трипод» // Машиностроение и компьютерные технологии.— 2009 .— № . 10.

7. Melnikov G. I. Mathematical models of nonlinear oscillations of mechanical systems with several degrees of freedom // Nonlinear Dynamics and Systems Theory, IET - 2017.-Vol. 17. — No. 4.-p. 369-375

( ) Иванов Сергей Евгеньевич ( sivanov@mail.ifmo.ru ), Зудилова Татьяна Викторовна ( zudilova@ifmo.spb.ru ),

Осетрова Ирина Станиславовна ( irina@ifmo.spb.ru ), Войтюк Татьяна Евгеньевна ( taire2006@yandex.ru ).

Журнал «Современная наука: актуальные проблемы теории и практики»

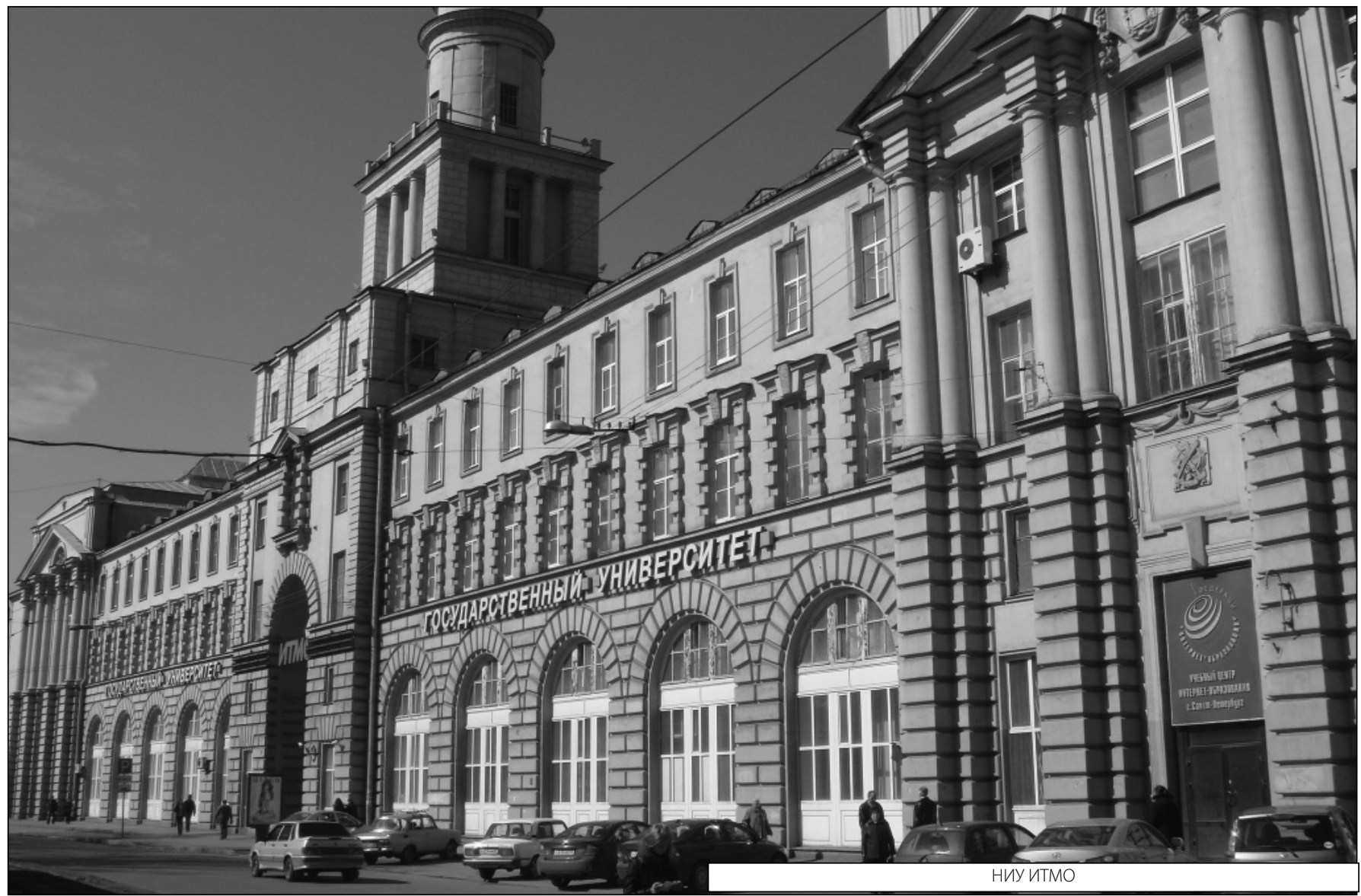

